\title{
Cooperating Distributed Grammar Systems of Finite Index Working in Hybrid Modes
}

\author{
Henning Fernau \\ Fachbereich 4-Abteilung Informatik \\ Universität Trier \\ D-54286 Trier, Germany \\ fernau@uni-trier.de
}

\author{
Rudolf Freund \\ Institut für Computersprachen \\ Technische Universität Wien \\ Favoritenstr. 9, A-1040 Wien, Austria \\ rudi@emcc. at
}

\author{
Markus Holzer \\ Institut für Informatik \\ Universität Gießen, \\ Arndtstraße 2, D-35392 Gießen, Germany \\ holzer@informatik.uni-giessen.de
}

\begin{abstract}
We study cooperating distributed grammar systems working in hybrid modes in connection with the finite index restriction in two different ways: firstly, we investigate cooperating distributed grammar systems working in hybrid modes which characterize programmed grammars with the finite index restriction; looking at the number of components of such systems, we obtain surprisingly rich lattice structures for the inclusion relations between the corresponding language families. Secondly, we impose the finite index restriction on cooperating distributed grammar systems working in hybrid modes themselves, which leads us to new characterizations of programmed grammars of finite index.
\end{abstract}

Keywords: CD grammar systems; finite index; hybrid modes; programmed grammars AMS MSC [2010] classification: 68Q42; 68Q45

\section{Introduction}

Cooperating distributed (CD) grammar systems first were introduced in [12] with motivations related to two-level grammars. Later, the investigation of CD grammar systems became a vivid area of research after relating CD grammar systems with Artificial Intelligence (AI) notions [2], such as multi-agent systems or blackboard models for problem solving. From this point of view, motivations for CD grammar systems can be summarized as follows: several grammars (agents or experts in the framework of AI), mainly consisting of rule sets (corresponding to scripts the agents have to obey to) are cooperating in order to work on a sentential form (representing their common work), finally generating terminal words (in this way solving the problem). The picture one has in mind is that of several grammars (mostly, these are simply classical context-free grammars called "components" in the theory of CD grammar systems) "sitting" around a table where there is lying the common workpiece, a sentential form. Some component takes this sentential form, works on it, i.e., it performs some derivation steps, and then returns it onto the table such that another component may continue the work.

In classical CD grammar systems, all components work in the same derivation mode. It is of course natural to alleviate this requirement, because it simply refers to different capabilities and working regulations of different experts in the original CD motivation. This leads to the notion of so-called hybrid CD grammar systems introduced by Mitrana and Păun in [13, 14]. We investigate internally hybrid derivation modes which partly allow for new characterizations of the external hybridizations explained above.

Z. Ésik and Z. Fülöp (Eds.): Automata and Formal Languages 2014 (AFL 2014) EPTCS 151, 2014, pp. 246-260 doi 10.4204/EPTCS.151.17

This work is dedicated to the public domain 
This paper belongs to a series of papers on hybrid modes in CD grammar systems: as predecessors, we mention that [6] introduces hybrid modes in CD array grammar systems as a natural specification tool for array languages and [10] investigates accepting CD grammar systems with hybrid modes; the two most relevant papers are [8, 9] where the most important aspects of internal and external mode hybridizations are discussed for the case of word languages.

Here, we will continue this line of research, focussing on the finite index restriction. The paper is organized as follows. In the next section, we introduce the necessary notions. In Section 3, we review important notions and results in connection with the finite index restriction. Section 4 is devoted to the study of internally hybrid CD grammar systems with the (explicit) restriction of being of finite index; we establish infinite hierarchies with respect to the number of components and the number of maximal derivation steps per component. In Section 5, we refine our previous analysis (published in [9]) showing characterizations of programmed grammars of finite index by several variants of (internally) hybrid CD grammar systems, also considering the number of grammar components as an additional descriptional complexity parameter. In the last section, we review our results again and give a prospect on possible future work.

\section{Definitions}

We assume the reader to be familiar with some basic notions of formal language theory and regulated rewriting, as contained in [15] and [4]. In particular, details on programmed grammars can be found there. In general, we have the following conventions: $\subseteq$ denotes inclusion, while $\subset$ denotes strict inclusion; the set of positive integers is denoted by $\mathbb{N}$. The empty word is denoted by $\lambda ;|\alpha|_{A}$ denotes the number of occurrences of the symbol $A$ in $\alpha$. We consider two languages $L_{1}, L_{2}$ to be equal if and only if $L_{1} \backslash\{\lambda\}=L_{2} \backslash\{\lambda\}$, and we simply write $L_{1}=L_{2}$ in this case. The families of languages generated by linear context-free and context-free grammars are denoted by $\mathscr{L}(\mathrm{LIN})$ and $\mathscr{L}(\mathrm{CF})$, respectively, and the family of finite languages is denoted by $\mathscr{L}(\mathrm{FIN})$. We attach $-\lambda$ in our notations for formal language classes if erasing rules are not permitted. Notice that we use bracket notations in order to express that the equation holds both in case of forbidding erasing rules and in the case of admitting erasing rules (consistently neglecting the contents between the brackets).

Next we introduce programmed grammars, a well-known concept in the area of regulated rewriting.

A programmed grammar is a septuple $G=(N, T, P, S, \Lambda, \sigma, \phi)$, where $N, T$, and $S \in N$ are the set of nonterminals, the set of terminals, and the start symbol, respectively. In the following we use $V_{G}$ to denote the set $N \cup T$, which is the complete working alphabet of the grammar. $P$ is the finite set of context-free rules $A \rightarrow z$ with $A \in N$ and $z \in V_{G}^{*}$, and $\Lambda$ is a finite set of labels (for the rules in $P$ ), such that $\Lambda$ can also be interpreted as a function which outputs a rule when being given a label; $\sigma$ and $\phi$ are functions from $\Lambda$ into the set of subsets of $\Lambda$. For $\left(x, r_{1}\right),\left(y, r_{2}\right)$ in $V_{G}^{*} \times \Lambda$ and $\Lambda\left(r_{1}\right)=(A \rightarrow z)$, we write $\left(x, r_{1}\right) \Rightarrow\left(y, r_{2}\right)$ if and only if either

1. $x=x_{1} A x_{2}, y=x_{1} z x_{2}$, and $r_{2} \in \sigma\left(r_{1}\right)$, or

2. $x=y$, the rule $A \rightarrow z$ is not applicable to $x$, and $r_{2} \in \phi\left(r_{1}\right)$.

In the latter case, the derivation step is performed in the so-called appearance checking mode. The set $\sigma\left(r_{1}\right)$ is called success field and the set $\phi\left(r_{1}\right)$ is called failure field of $r_{1}$. As usual, the reflexive transitive closure of $\Rightarrow$ is denoted by $\Longrightarrow$. The language generated by $G$ is defined as

$$
L(G)=\left\{w \in T^{*} \mid\left(S, r_{1}\right) \Longrightarrow^{*}\left(w, r_{2}\right) \text { for some } r_{1}, r_{2} \in \Lambda\right\}
$$


The family of languages generated by $[\lambda$-free] programmed grammars containing only context-free rules is denoted by $\mathscr{L}(\mathrm{P}, \mathrm{CF}[-\lambda]$, ac $)$. When no appearance checking features are involved, i.e., $\phi(r)=\emptyset$ for each label $r \in \Lambda$, we obtain the family $\mathscr{L}(\mathrm{P}, \mathrm{CF}[-\lambda])$.

Finally, we now define cooperating distributed (CD) and hybrid cooperating distributed (HCD) grammar systems.

A $C D$ grammar system of degree $n$, with $n \geq 1$, is an $(n+3)$-tuple $G=\left(N, T, S, P_{1}, P_{2}, \ldots, P_{n}\right)$, where $N, T$ are disjoint alphabets of nonterminal and terminal symbols, respectively, $S \in N$ is the start symbol, and $P_{1}, \ldots, P_{n}$ are finite sets of rewriting rules over $N \cup T$. Throughout this paper, we consider only regular, linear context-free, and context-free rewriting rules. For $x, y \in(N \cup T)^{*}$ and $1 \leq i \leq n$, we write $x \Longrightarrow_{i} y$ if and only if $x=x_{1} A x_{2}, y=x_{1} z x_{2}$ for some $A \rightarrow z \in P_{i}$. Hence, subscript $i$ refers to the component to be used. Accordingly, $x \Longrightarrow_{i}^{m} y$ denotes an $m$-step derivation using component number $i$, where $x \Longrightarrow_{i}^{0} y$ if and only if $x=y$.

We define the classical basic modes $B=\{*, t\} \cup\{\leq k,=k, \geq k \mid k \in \mathbb{N}\}$ and let

$$
D=B \cup\{(\geq k \wedge \leq \ell) \mid k, \ell \in \mathbb{N}, k \leq \ell\} \cup\{(t \wedge \leq k),(t \wedge=k),(t \wedge \geq k) \mid k \in \mathbb{N}\} .
$$

For $f \in D$ we define the relation $\Longrightarrow_{i}^{f}$ by

$$
x \Longrightarrow_{i}^{f} y \Longleftrightarrow \exists m \geq 0:\left(x \Longrightarrow_{i}^{m} y \wedge P(f, m, i, y)\right),
$$

where $P$ is a predicate defined as follows (let $k \in \mathbb{N}$ and $f_{1}, f_{2} \in B$ ):

\begin{tabular}{l|c} 
predicate & definition \\
\hline \hline$P(=k, m, i, y)$ & $m=k$ \\
$P(\leq k, m, i, y)$ & $m \leq k$ \\
$P(\geq k, m, i, y)$ & $m \geq k$ \\
$P(*, m, i, y)$ & $m \geq 0$ \\
$P(t, m, i, y)$ & $\neg \exists z\left(y \Longrightarrow_{i} z\right)$ \\
$P\left(\left(f_{1} \wedge f_{2}\right), m, i, y\right)$ & $P\left(f_{1}, m, i, y\right) \wedge P\left(f_{2}, m, i, y\right)$
\end{tabular}

Observe that not every combination of modes as introduced above is a genuinely hybrid mode. For example, the $(\geq k \wedge \leq k)$-mode is just another notation for the $=k$-mode. Especially, $*$ may be used as a "don't care" in our subsequent notations, since $P\left(\left(* \wedge f_{2}\right), m, i, y\right)$ if and only if $P\left(f_{2}, m, i, y\right)$.

If each component of a CD grammar system may work in a different mode, then we get the notion of an (externally) hybrid $C D$ (HCD) grammar system of degree $n$, with $n \geq 1$, which is an $(n+3)$-tuple $G=\left(N, T, S,\left(P_{1}, f_{1}\right),\left(P_{2}, f_{2}\right), \ldots,\left(P_{n}, f_{n}\right)\right)$, where $N, T, S, P_{1}, \ldots, P_{n}$ are as in a CD grammar system, and $f_{i} \in D$, for $1 \leq i \leq n$. Thus, we can define the language generated by a HCD grammar system as:

$$
\begin{aligned}
& L(G):=\left\{w \in T^{*} \mid S \Rightarrow \Rightarrow_{i_{1}}^{f_{i_{1}}} w_{1} \Rightarrow_{i_{2}}^{f_{i_{2}}} \ldots \Rightarrow_{i_{m-1}}^{f_{i_{m-1}}} w_{m-1} \Rightarrow_{i_{m}}^{f_{i_{m}}} w_{m}=w\right. \\
& \text { with } \left.m \geq 1,1 \leq i_{j} \leq n \text {, and } 1 \leq j \leq m\right\}
\end{aligned}
$$

If $F \subseteq D$ and $X \in\{\mathrm{LIN}, \mathrm{CF}\}$, then the family of languages generated by [ $\lambda$-free] HCD grammar systems with degree at most $n$ using rules of type $X$, each component working in one of the modes contained in $F$, is denoted by $\mathscr{L}\left(\mathrm{HCD}_{n}, X[-\lambda], F\right)$. In a similar way, we write $\mathscr{L}\left(\mathrm{HCD}_{\infty}, X[-\lambda], F\right)$ when the number of components is not restricted. If $F$ is a singleton $\{f\}$, we simply write $\mathscr{L}\left(\mathrm{CD}_{n}, X[-\lambda], f\right)$, where $n \in \mathbb{N} \cup\{\infty\}$; additionally, we write $L_{f}(G)$ instead of $L(G)$ to denote the language generated by the CD grammar system $G$ in the mode $f$.

The following example is taken from [8, Theorem 24], as we need this language in the following of this paper. This should also help to clarify our definitions. 
Example 1 The non-context-free language $L=\left\{a_{1}^{n} a_{2}^{n} \ldots a_{k+1}^{n} \mid n \geq 1\right\}$ can be generated by the $C D$ grammar system $G=\left(N, T, S_{1}, P_{1}, P_{2}\right)$, where $P_{1}, P_{2}$ work in the $(t \wedge \geq k)$-mode, $k \geq 2$. For both components, we take $N=\left\{S_{i}, A_{i}, A_{i}^{\prime}, \mid 1 \leq i \leq k\right\}$ as nonterminal alphabet and $T=\left\{a_{1}, \ldots, a_{k+1}\right\}$ as terminal alphabet. The components $P_{1}$ and $P_{2}$ are defined as follows:

$$
\begin{aligned}
P_{1}= & \left\{S_{i} \rightarrow S_{i+1} \mid 1 \leq i<k\right\} \cup\left\{S_{k} \rightarrow A_{1} \cdots A_{k}\right\} \cup \\
& \left\{A_{i}^{\prime} \rightarrow A_{i} \mid 1 \leq i \leq k\right\} \text { and } \\
P_{2}= & \left\{A_{i} \rightarrow a_{i} A_{i}^{\prime} \mid 1 \leq i \leq k-1\right\} \cup\left\{A_{k} \rightarrow a_{k} A_{k}^{\prime} a_{k+1}\right\} \cup \\
& \left\{A_{i} \rightarrow a_{i} \mid 1 \leq i \leq k-1\right\} \cup\left\{A_{k} \rightarrow a_{k} a_{k+1}\right\}
\end{aligned}
$$

Then we have $L(G)=L$, since every derivation of $G$ leading to a terminal word is of the form

$$
S_{1} \quad{ }_{1}^{=k} A_{1} \ldots A_{k} \quad \cdots \quad \Longrightarrow={ }_{2}^{=k} a_{1}^{n} \ldots a_{k}^{n} a_{k+1}^{n},
$$

where the intermediate steps are of the form

$$
a_{1}^{i} A_{1} \ldots a_{k}^{i} A_{k} a_{k+1}^{i} \Longrightarrow{ }_{2}^{=k} a_{1}^{i+1} A_{1}^{\prime i+1}{ }_{k} A_{k}^{\prime i+1}{ }_{k+1} \Longrightarrow_{1}^{=k} a_{1}^{i+1} A_{1} \ldots a_{k}^{i+1} A_{k} a_{k+1}^{i+1}
$$

if a non-vanishing number of occurrences of $A_{i}^{\prime}$ less than $k$ is obtained by using $P_{2}$ then neither $P_{1}$ nor $P_{2}$ can perform $k$ derivation steps any more. Hence, $G$ generates $L$.

The same grammar system, viewed as a $\left(\mathrm{CD}_{2}, \mathrm{CF},(t \wedge=k)\right)$ grammar system, generates $L$, too.

\section{The Finite Index Restriction}

The finite index restriction is defined as follows: let $G$ be an arbitrary grammar type (from those discussed in Section 2) and let $N, T$, and $S \in N$ be its nonterminal alphabet, terminal alphabet, and axiom, respectively. For a derivation

$$
D: S=w_{1} \Longrightarrow w_{2} \Longrightarrow \cdots \Longrightarrow w_{n}=w \in T^{*}
$$

according to $G$, we set $\operatorname{ind}(D, G)=\max \left\{\left|w_{i}\right|_{N} \mid 1 \leq i \leq n\right\}$. In the case of programmed grammars we assume to have a derivation of the form

$$
D:\left(S, r_{1}\right)=\left(w_{1}, r_{1}\right) \Longrightarrow\left(w_{2}, r_{2}\right) \Longrightarrow \cdots \Longrightarrow\left(w_{n}, r_{n}\right)=\left(w, r_{n}\right) \in T^{*} \times \Lambda .
$$

For $w \in T^{*}$, we define $\operatorname{ind}(w, G)=\min \{\operatorname{ind}(D, G) \mid D$ is a derivation for $w$ in $G\}$. The index of grammar $G$ is defined as $\operatorname{ind}(G)=\sup \{\operatorname{ind}(w, G) \mid w \in L(G)\}$. For a language $L$ in the family $\mathscr{L}(\mathrm{X})$ of languages generated by grammars of type $\mathrm{X}$, we define $\operatorname{ind}_{\mathrm{X}}(L)=\inf \{\operatorname{ind}(G) \mid L(G)=L$ and $G$ is of type $\mathrm{X}\}$. For a family $\mathscr{L}(\mathrm{X})$, we set

$$
\begin{aligned}
\mathscr{L}_{n}(\mathrm{X}) & =\left\{L \mid L \in \mathscr{L}(\mathrm{X}) \text { and } \operatorname{ind}_{\mathrm{X}}(L) \leq n\right\} \quad \text { for } n \in \mathbb{N}, \text { and } \\
\mathscr{L}_{f i n}(\mathrm{X}) & =\bigcup_{n \geq 1} \mathscr{L}_{n}(\mathrm{X}) .
\end{aligned}
$$

It is well-known that the class of programmed languages of index $m$ can be characterized in various ways, compare, e.g., [4, 11, 16]. Especially, normal forms are available. For the reader's convenience, we quote [9, Theorem 9] in the following, since we will use it to give a sharpened and broadened version of [3, Theorem 3.26], which leads us to new characterizations of the classes $\mathscr{L}_{m}(\mathrm{P}, \mathrm{CF})$ and $\mathscr{L}_{\text {fin }}(\mathrm{P}, \mathrm{CF})$. 
Theorem 1 For every $(\mathrm{P}, \mathrm{CF}, \mathrm{ac})$ grammar $G=(N, T, P, S, \Lambda, \sigma, \phi)$ whose generated language is of index $n \in \mathbb{N}$, there exists an equivalent $\left(\mathrm{P}, \mathrm{CF}\right.$, ac) grammar $G^{\prime}=\left(N^{\prime}, T, P^{\prime}, S^{\prime}, \Lambda, \sigma^{\prime}, \phi^{\prime}\right)$ whose generated language is also of index $n$ and which satisfies the following three properties:

1. There exists a special start production with a unique label $p_{0}$, which is the only production where the start symbol $S^{\prime}$ appears.

2. There exists a function $f: \Lambda^{\prime} \rightarrow \mathbb{N}_{0}^{N^{\prime}}$ such that, if $S^{\prime} \Longrightarrow^{*} v \Longrightarrow_{p} w$ is a derivation in $G^{\prime}$, then $(f(p))(A)=|v|_{A}$ for every nonterminal $A$.

3. If $D: S^{\prime}=v_{0} \Longrightarrow r_{1} v_{1} \Longrightarrow r_{2} v_{2} \cdots \Longrightarrow r_{m} v_{m}=w$ is a derivation in $G^{\prime}$ then, for every $v_{i}, 0 \leq i \leq m$, and every nonterminal $A,\left|v_{i}\right|_{A} \leq 1$. In other words, every nonterminal occurs at most once in any derivable sentential form.

Moreover, we may assume that either $G^{\prime}$ is a $(\mathrm{P}, \mathrm{CF})$ grammar, i.e., we have $\phi^{\prime}=\emptyset$, or that $G^{\prime}$ is a $(\mathrm{P}, \mathrm{CF}, \mathrm{ut})$ grammar, i.e., we have $\phi^{\prime}=\sigma^{\prime}$.

In the following, we will refer to a grammar satisfying the three conditions listed above as nonterminal separation form (NSF).

Theorem 1 shows that, in contrast to the general case, where $\mathscr{L}(\mathrm{P}, \mathrm{CF}, \mathrm{ac}) \supset \mathscr{L}(\mathrm{P}, \mathrm{CF})$, the appearance checking feature does not increase the generative power of programmed grammars if the finite index restriction is imposed; especially we have $\mathscr{L}_{m}(\mathrm{P}, \mathrm{CF}[-\lambda]$, ac $)=\mathscr{L}_{m}(\mathrm{P}, \mathrm{CF}[-\lambda])$.

Recall that we have shown in [9, Theorem 30] the following link between hybrid CDGS and the finite index restriction on programmed grammars.

Theorem 2 Let $\ell \in \mathbb{N}$ and $\Delta \in\{\leq,=\}$. Then we have:

$$
\begin{aligned}
\mathscr{L}\left(\mathrm{HCD}_{\infty}, \mathrm{CF}[-\lambda],\{(t \wedge \Delta k) \mid k \geq 1\}\right) & =\bigcup_{k \in \mathbb{N}} \mathscr{L}\left(\mathrm{CD}_{\infty}, \mathrm{CF}[-\lambda],(t \wedge \Delta k)\right) \\
& =\mathscr{L}\left(\mathrm{CD}_{\infty}, \mathrm{CF}[-\lambda],(t \wedge \Delta l)\right) \\
& =\mathscr{L}\left(\mathrm{CD}_{\infty}, \mathrm{CF}[-\lambda],(t \wedge \Delta 1)\right) \\
& =\mathscr{L}_{\text {fin }}(\mathrm{P}, \mathrm{CF}[-\lambda], \mathrm{ac}) .
\end{aligned}
$$

Unfortunately, our proof did not bound the number of components of the CD grammar system. This is not just a coincidence, as we will see in this paper.

\section{Infinite Hierarchies for CD Grammar Systems Working in Hybrid Modes}

Our task will be the study of the language families $\mathscr{L}\left(\mathrm{CD}_{n}, \mathrm{CF}[-\lambda],(t \wedge \Delta k)\right)$ for different $n, k \in \mathbb{N}$ and $\Delta \in\{\leq,=\}$. First we give some characterizations of well-known language families, namely the family of finite languages and the family of linear languages.

Lemma 3 For every $k \in \mathbb{N}$, and $\Delta \in\{\leq,=\}$, we have

$$
\mathscr{L}(\mathrm{FIN})=\mathscr{L}\left(\mathrm{CD}_{1}, \mathrm{CF}[-\lambda],(t \wedge \Delta k)\right) .
$$


Proof. Since we have only one component, by definition of the $(t \wedge \Delta k)$-mode, every derivation has length at most $k$, so that we only get finite languages. If $L=\left\{w_{1}, w_{2}, \ldots, w_{m}\right\} \subseteq T^{*}$ is some finite language, then the grammar $G=(\{S\} \times\{1, \ldots, k\}, T,(S, 1), P)$ with

$$
\left.P=\{(S, i) \rightarrow(S, i+1) \mid 1 \leq i<k\} \cup\left\{(S, k) \rightarrow w_{j} \mid 1 \leq j \leq m\right\}\right)
$$

generates $L$.

Now we turn our attention to CD grammar systems with two components working in the $(t \wedge \Delta 1)$ mode for $\Delta \in\{\leq,=\}$.

Lemma 4 For $\Delta \in\{\leq,=\}$ we have $\mathscr{L}(\mathrm{LIN})=\mathscr{L}\left(\mathrm{CD}_{2}, \mathrm{CF}[-\lambda],(t \wedge \Delta 1)\right)$.

Proof. Let $L$ be generated by the linear grammar $G=(N, T, S, P)$. Grammar $G$ is simulated by the CD grammar system $G^{\prime}=\left(N \cup N^{\prime}, T, S, P_{1}, P_{2}\right)$ where $N^{\prime}$ contains primed versions of the nonterminals of $G$, set $P_{1}$ contains colouring unit productions $B \rightarrow B^{\prime}$ for every nonterminal $B \in N$, and $P_{2}$ contains, for every production $A \rightarrow w \in P$, a production $A^{\prime} \rightarrow w$. The simulation of $G$ by $G^{\prime}$ proceeds by applying $P_{2}$ and $P_{1}$ in sequence until the derivation stops.

On the other hand, it is easy to see that no sentential form generated by some $\left(\mathrm{CD}_{2}, \mathrm{CF}[-\lambda],(t \wedge\right.$ $\Delta 1)$ )-system (eventually leading to a terminal string) can contain more than one nonterminal. Otherwise, we must have applied a production $A \rightarrow w$ of say the first component, where $w$ contains at least two nonterminals. All nonterminals occurring in $w$ cannot be processed further by the first component, since otherwise it violates the $(t \wedge \Delta 1)$-mode restriction. But nearly the same argument applies to the second component, too: it can only process at most one of the nonterminals just introduced. Hence, no terminal string is derivable in this way.

Therefore, one can omit all productions containing more than one nonterminal on their right-hand sides, so that there are only linear rules left. Furthermore, one can also omit all productions in a component containing a nonterminal as its right-hand side which occurs also as the left-hand side of the originally given component as this would lead to more than one derivation step in the same component. Now, one can put all remaining productions together yielding the rule set of a simulating linear grammar.

In the general case, i.e., two components working together in the $(t \wedge \Delta k)$-mode, for $k \in \mathbb{N}$ and $\Delta \in\{\leq,=\}$, we first give some lower bounds.

Theorem 5 Let $k \in \mathbb{N}, \Delta \in\{\leq,=\}$. Then we have:

1. $\mathscr{L}(\mathrm{LIN})=\mathscr{L}_{1}(\mathrm{CF})=\mathscr{L}\left(\mathrm{CD}_{2}, \mathrm{CF}[-\lambda],(t \wedge \Delta 1)\right)$;

2. $\mathscr{L}_{k}(\mathrm{CF}) \subseteq \mathscr{L}\left(\mathrm{CD}_{2}, \mathrm{CF}[-\lambda],(t \wedge \Delta k)\right)$ for $k \geq 1$, and

3. $\mathscr{L}_{k}(\mathrm{CF}) \subset \mathscr{L}\left(\mathrm{CD}_{2}, \mathrm{CF}[-\lambda],(t \wedge=k)\right)$ for $k>1$;

4. $\mathscr{L}_{\text {fin }}(\mathrm{CF}) \subset \bigcup_{k \in \mathbb{N}} \mathscr{L}\left(\mathrm{CD}_{2}, \mathrm{CF}[-\lambda],(t \wedge=k)\right)$.

\section{Proof.}

1. It is easy to see that $\mathscr{L}(\mathrm{LIN})=\mathscr{L}_{1}(\mathrm{CF})$. Hence, this statement is equivalent to the assertion of the previous lemma.

2. Let $G=(N, T, S, P)$ be a context-free grammar of index $k$. Without loss of generality, we assume that every nonterminal occurs as the left-hand side of some production in $P$. Let $N^{\prime}$ be the set of primed nonterminal symbols. Grammar $G$ is simulated by the $\mathrm{CD}$ grammar system 
$G^{\prime}=\left(N \cup N^{\prime}, T, S, P_{1}, P_{2}\right)$, where $P_{1}$ contains colouring unit productions $B \rightarrow B^{\prime}$, and $B \rightarrow B$ for every nonterminal $B \in N$, and $P_{2}$, for every production $A \rightarrow w \in P$, contains productions $A^{\prime} \rightarrow w$ and $A^{\prime} \rightarrow A^{\prime}$. The unit productions $B \rightarrow B$ in $P_{1}$ and $A^{\prime} \rightarrow A^{\prime}$ in $P_{2}$ guarantee that at most $k$ nonterminals can occur in any sentential form that can be derived in $G^{\prime}$.

3. A separating example was already explained in Example 1, there the languages $\left\{a_{1}^{n} a_{2}^{n} \ldots a_{k+1}^{n} \mid\right.$ $n \geq 1\}$ was shown to be in $\mathscr{L}\left(\mathrm{CD}_{2}, \mathrm{CF}[-\lambda],(t \wedge=k)\right)$ for $k \geq 2$, but obviously these languages are not context-free.

4. Follows from 3.

Unfortunately, we do not know whether the inclusion

$$
\mathscr{L}_{k}(\mathrm{CF}) \subseteq \mathscr{L}\left(\mathrm{CD}_{2}, \mathrm{CF}[-\lambda],(t \wedge \leq k)\right)
$$

in the previous theorem is strict or not. By the prolongation technique introduced in $[\overline{8}]$, we know that the classes $\mathscr{L}\left(\mathrm{CD}_{n}, \mathrm{CF}[-\lambda],(t \wedge \Delta k)\right)$, for $\Delta \in\{\leq,=\}$ form a prime number lattice, i.e.,

$$
\mathscr{L}\left(\mathrm{CD}_{n}, \mathrm{CF}[-\lambda],(t \wedge \Delta k)\right) \subseteq \mathscr{L}\left(\mathrm{CD}_{n}, \mathrm{CF}[-\lambda],(t \wedge \Delta \ell \cdot k) \quad \text { for } \ell \in \mathbb{N},\right.
$$

with the least element $\mathscr{L}\left(\mathrm{CD}_{n}, \mathrm{CF}[-\lambda],(t \wedge \Delta 1)\right)$. This prolongation technique is based on the simple idea to "slow down" a derivation using $A \rightarrow w$ of the original CDGS by intercalating productions of the form $A \rightarrow A^{\prime}, A^{\prime} \rightarrow A^{\prime \prime}, \ldots, A^{(j)} \rightarrow w$ within the simulating CDGS. It will be used on several occasions in this paper. Obviously, we also have the trivial inclusions

$$
\mathscr{L}\left(\mathrm{CD}_{n}, \mathrm{CF}[-\lambda],(t \wedge \Delta k)\right) \subseteq \mathscr{L}\left(\mathrm{CD}_{n+1}, \mathrm{CF}[-\lambda],(t \wedge \Delta k)\right) \text { for } \Delta \in\{\leq,=\} .
$$

The question arises whether all these hierarchies are strict. At least we will be able to show that both with respect to $k$ - for a fixed number of components $n$ - as well as with respect to the number of components $n$ - for a fixed derivation mode $(t \wedge \Delta k), \Delta \in\{\leq,=\}-$ we obtain infinite hiearachies. In order to prove these hierarchies, we show some general theorems relating the number of components and the bound of the number of symbols to be rewritten by one component with the finite index of a simulating programmed grammar.

Theorem 6 Let $n, k \in \mathbb{N}$ and $\Delta \in\{\leq,=\}$. Then, we have

$$
\mathscr{L}\left(\mathrm{CD}_{n}, \mathrm{CF}[-\lambda],(t \wedge \Delta k)\right) \subseteq \mathscr{L}_{n \cdot k}(\mathrm{P}, \mathrm{CF}[-\lambda], a c) .
$$

Proof. Let $G=\left(N, T, S, P_{1}, P_{2}, \ldots, P_{n}\right)$ be a $\mathrm{CD}$ grammar system working in the $(t \wedge=k)$-mode. Let $P_{i}=\left\{A_{i j} \rightarrow w_{i j} \mid 1 \leq j \leq N(i)\right\}$. $G$ can be simulated by the programmed grammar $G^{\prime}=(N \cup$ $\{F\}, T, S, P, \Lambda, \sigma, \phi)$, with label-set

$$
\begin{aligned}
\Lambda= & \{(i, j, \kappa) \mid 1 \leq i \leq n, 1 \leq j \leq N(i), 1 \leq \kappa \leq k\} \\
& \cup\{(i, j) \mid 1 \leq i \leq n, 1 \leq j \leq N(i)\} .
\end{aligned}
$$

Now, let $1 \leq i \leq n, 1 \leq j \leq N(i), 1 \leq \kappa \leq k$. Then, we set $\Lambda((i, j, \kappa))=A_{i j} \rightarrow w_{i j}$, success field $\sigma((i, j, \kappa))=\left\{\left(i, j^{\prime}, \kappa+1\right) \mid 1 \leq j^{\prime} \leq N(i)\right\}$, if $\kappa<k$, and $\sigma((i, j, k))=\{(i, 1)\}$, and failure field $\phi((i, j, \kappa))=\emptyset$. Moreover, we let $\Lambda((i, j))=A_{i j} \rightarrow F$, failure field $\phi((i, j))=\{(i, j+1)\}$ if $j<N(i)$, $\phi((i, N(i)))=\left\{\left(i^{\prime}, j^{\prime}, 1\right) \mid 1 \leq i^{\prime} \leq n, 1 \leq j^{\prime} \leq N\left(i^{\prime}\right)\right\}$, and success field $\sigma((i, j))=\emptyset$. 
An application of $P_{i}$ is simulated by a sequence of productions labeled with

$$
\left(i, j_{1}, 1\right), \ldots,\left(i, j_{k}, k\right),(i, 1), \ldots,(i, N(i)) .
$$

In each such sequence, at most $k$ symbols can be processed. Since there are $n$ sets of productions $P_{i}$, only sentential forms containing at most $n \cdot k$ nonterminals can hope for termination. Therefore, the simulating programmed grammar has index at most $n \cdot k$, which can be seen by induction.

The $(t \wedge \leq k)$-mode case can be treated in a similar way: we just define $\phi((i, j, \kappa))$ to equal $\sigma((i, j, \kappa))$ instead of taking $\phi((i, j, \kappa))=\emptyset$.

Before we can establish the infinite hierarchies for the families $\mathscr{L}\left(\mathrm{CD}_{n}, \mathrm{CF}[-\lambda],(t \wedge \Delta k)\right)$ with respect to $n$ and $k$, respectively, we need the following theorem shown in [4, page 160, Theorem 3.1.7]:

Theorem $7 S_{n+1}=\left\{b\left(a^{i} b\right)^{2 \cdot(n+1)} \mid i \geq 1\right\} \in \mathscr{L}_{n+1}(\mathrm{P}, \mathrm{CF}) \backslash \mathscr{L}_{n}(\mathrm{P}, \mathrm{CF})$ for all $n \in \mathbb{N}$.

These separating languages can also be generated by CD grammar systems working in the internally hybrid modes $(t \wedge=k+1)$ and $(t \wedge \leq k+1)$ :

Theorem 8 Let $n, k \in \mathbb{N}$.

1. $S_{n \cdot k} \in \mathscr{L}\left(\mathrm{CD}_{n+1}, \mathrm{CF}-\lambda,(t \wedge=k+1)\right)$, i.e., $S_{n \cdot k}$ can be generated by a $C D$-grammar system with $n+1$ context-free components, without erasing productions, working in the $(t \wedge=k+1)$-mode.

2. $S_{n \cdot k} \in \mathscr{L}\left(\mathrm{CD}_{2 n+1}, \mathrm{CF}-\lambda,(t \wedge \leq k+1)\right)$, i.e., $S_{n \cdot k}$ can be generated by $C D$-grammar system with $2 \cdot n+1$ context-free components, without erasing productions, working in the $(t \wedge \leq k+1)$-mode.

Proof. We first construct a CD grammar system

$$
G=\left(N,\{a, b\},(S, 0), P_{0}, P_{1,1} \cup P_{1,2}, \ldots, P_{n, 1} \cup P_{n, 2}\right)
$$

working in the $(t \wedge=k+1)$-mode generating language $S_{n \cdot k}$. Let

$$
\begin{aligned}
N= & \{(S, i) \mid 0 \leq i \leq n\} \\
\cup & \left\{\left(q_{i}, 0\right),\left(q_{i}, 1\right) \mid 1 \leq i \leq n\right\} \\
\cup & \left\{\left(t_{i}, j\right),\left(t_{i}^{\prime}, j\right) \mid 1 \leq i \leq n, 0 \leq j \leq k\right\} \\
\cup & \left\{\left(A_{i}, 0\right),\left(A_{i}, 1\right) \mid 1 \leq i \leq k \cdot n\right\}
\end{aligned}
$$

and let the set of productions be as follows:

$$
\begin{aligned}
& P_{0}=\left\{(S, 0) \rightarrow(S, 1),(S, k) \rightarrow\left(q_{1}, 0\right)\left(A_{1}, 0\right) b\left(A_{2}, 0\right) b \ldots b\left(A_{n \cdot k}, 0\right) b\right\} \\
& \cup\{(S, i) \rightarrow(S, i+1) \mid 1 \leq i<k\} \\
& \cup \quad\left\{\left(A_{i}, 1\right) \rightarrow\left(A_{i}, 0\right) \mid 1 \leq i \leq n \cdot k\right\} \\
& \cup \quad\left\{\left(q_{i}, 1\right) \rightarrow\left(q_{i+1}, 0\right) \mid 1 \leq i<n\right\} \cup\left\{\left(q_{n}, 1\right) \rightarrow\left(q_{1}, 0\right),\left(q_{n}, 1\right) \rightarrow\left(t_{1}, 0\right)\right\} \\
& \cup \quad\left\{\left(t_{i}^{\prime}, j\right) \rightarrow\left(t_{i}^{\prime}, j+1\right) \mid 1 \leq i \leq n, 0 \leq j<k\right\} \\
& \cup \quad\left\{\left(t_{i}^{\prime}, k\right) \rightarrow\left(t_{i+1}, 0\right) \mid 1 \leq i<n\right\} \cup\left\{\left(t_{n}^{\prime}, k\right) \rightarrow b\right\} .
\end{aligned}
$$

For every $1 \leq i \leq n$ let $P_{i}=P_{i, 1} \cup P_{i, 2}$ where

$$
\begin{aligned}
P_{i, 1} & =\left\{\left(q_{i}, 0\right) \rightarrow\left(q_{i}, 1\right)\right\} \\
& \cup\left\{\left(A_{j}, 0\right) \rightarrow a\left(A_{j}, 1\right) a \mid(i-1) \cdot k \leq j \leq i \cdot k\right\} \quad \text { and } \\
P_{i, 2} & =\left\{\left(t_{i}, 0\right) \rightarrow\left(t_{i}^{\prime}, 0\right)\right\} \\
& \cup\left\{\left(A_{j}, 0\right) \rightarrow a b a \mid(i-1) \cdot k \leq j \leq i \cdot k\right\} .
\end{aligned}
$$


The only way to start the derivation is to use $P_{0}$ obtaining the word

$$
\left(q_{1}, 0\right)\left(A_{1}, 0\right) b\left(A_{2}, 0\right) b \ldots b\left(A_{n \cdot k}, 0\right) b .
$$

To continue the derivation one always has to apply $P_{i}$ and $P_{0}$ in sequence: $P_{i}$ is only successfully applicable to a sentential form beginning with a letter $\left(q_{i}, 0\right)$ or $\left(t_{i}, 0\right)$, and $P_{0}$ is only successfully applicable to a sentential form beginning with a letter $\left(q_{i}, 1\right)$ or $\left(t_{i}^{\prime}, 0\right)$.

Assume we have a sentential form starting with letter $\left(q_{i}, 0\right)$, then rules from $P_{i}$ replace exactly $k$ occurrences of nonterminals $\left(A_{j}, 0\right)$, for $(i-1) \cdot k \leq j \leq i \cdot k$, by $a\left(A_{j}, 1\right) a$ or $a b a$, respectively, and the label is changed to $\left(q_{i}, 1\right)$. Now applying the corresponding rules from $P_{0}$ (the only way to continue), the derivation would block if at least one of the symbols $\left(A_{j}, 0\right)$ from the previous step were replaced by $a b a$. This ensures that all previously used productions are non-terminating. In case the sentential from starts with a letter $\left(t_{i}, 0\right)$, then an application of $P_{i}$ followed by $P_{0}$ checks whether the terminating rules form $P_{i}$ were all used or not.

Thus, starting with the word $\left(q_{1}, 0\right)\left(A_{1}, 0\right) b\left(A_{2}, 0\right) b \ldots b\left(A_{n \cdot k}, 0\right) b$, one cycle, i.e., an application of $P_{1}, P_{0}, P_{2}, P_{0}, \ldots, P_{n}$, and $P_{0}$ leads to

$$
\left(q_{1}, 0\right) a\left(A_{1}, 0\right) a b a\left(A_{2}, 0\right) a b \ldots b a\left(A_{n \cdot k}, 0\right) a b,
$$

and in general, running the cycle $\ell$ times, to the word

$$
\left(q_{1}, 0\right) a^{\ell}\left(A_{1}, 0\right) a^{\ell} b a^{\ell}\left(A_{2}, 0\right) a^{\ell} b \ldots b a^{\ell}\left(A_{n \cdot k}, 0\right) a^{\ell} b .
$$

Note that in the last application of $P_{0}$ we could have taken the rule $\left(q_{n}, 1\right) \rightarrow\left(t_{1}, 0\right)$ in order to terminate the derivation process after having finished the next cycle. After that cycle the grammar $G$ has generated the word $b\left(a^{\ell+1} b\right)^{2 n \cdot k}$.

In case of the $(t \wedge \leq k+1)$-mode, we use the same construction as above, but now treat each $P_{i, j}$, for $1 \leq i \leq n$ and $1 \leq j \leq 2$, as an independent component of the grammar. This gives the bound $2 \cdot n+1$ on the number of components.

Now we are ready to investigate the families $\mathscr{L}\left(\mathrm{CD}_{n}, \mathrm{CF}[-\lambda],(t \wedge \Delta k)\right)$ in more detail. First, let the number of components $n$ be fixed.

Corollary 9 Let $\Delta \in\{\leq,=\}$ and $n \in \mathbb{N}$ be fixed. The hierarchy of the families of languages $\mathscr{L}_{k}:=$ $\mathscr{L}\left(\mathrm{CD}_{n}, \mathrm{CF}[-\lambda],(t \wedge \Delta k)\right)$ with respect to $k \in \mathbb{N}$ is infinite, i.e., for every $k \in \mathbb{N}$ there exists an $m \in \mathbb{N}$, $m>k$, such that $\mathscr{L}_{k} \subset \mathscr{L}_{m}$.

Proof. We first consider the $(t \wedge=k)$-mode. By the preceding theorem we have that

$$
S_{n \cdot m} \in \mathscr{L}\left(\mathrm{CD}_{n+1}, \mathrm{CF}[-\lambda],(t \wedge=m+1)\right) .
$$

On the other hand, $S_{n \cdot m} \notin \mathscr{L}_{n \cdot m-1}(\mathrm{P}, \mathrm{CF})$ according to Theorem 7 and, because of

$$
\mathscr{L}\left(\mathrm{CD}_{n+1}, \mathrm{CF}[-\lambda],(t \wedge=k)\right) \subseteq \mathscr{L}_{(n+1) \cdot k}(\mathrm{P}, \mathrm{CF})
$$

according to Theorem 6, $S_{n \cdot m}$ therefore cannot belong to $\mathscr{L}\left(\mathrm{CD}_{n+1}, \mathrm{CF}[-\lambda],(t \wedge=k)\right)$, provided $n \cdot m-$ $1 \geq(n+1) \cdot k$, i.e., $m \geq \frac{n+1}{n} k+\frac{1}{n}$.

For the $(t \wedge \leq k)$-mode, we can argue in a similar way: By the preceding theorem we have that

$$
S_{n \cdot m} \in \mathscr{L}\left(\mathrm{CD}_{2 n+1}, \mathrm{CF}[-\lambda],(t \wedge \leq m+1)\right)
$$


and therefore $S_{n \cdot m} \in \mathscr{L}\left(\mathrm{CD}_{2 n+2}, \mathrm{CF}[-\lambda],(t \wedge \leq m+1)\right)$, too. On the other hand, $S_{n \cdot m} \notin \mathscr{L}_{n \cdot m-1}(\mathrm{P}, \mathrm{CF})$ according to Theorem 7 and, because of

$$
\mathscr{L}\left(\mathrm{CD}_{2 n+1}, \mathrm{CF}[-\lambda],(t \wedge \leq k)\right) \subseteq \mathscr{L}_{(2 n+1) \cdot k}(\mathrm{P}, \mathrm{CF}) \subseteq \mathscr{L}_{(2 n+2) \cdot k}(\mathrm{P}, \mathrm{CF})
$$

according to Theorem 6, $S_{n \cdot m}$ therefore cannot belong to $\mathscr{L}\left(\mathrm{CD}_{2 n+1}, \mathrm{CF}[-\lambda],(t \wedge \leq k)\right) \cap$ $\mathscr{L}\left(\mathrm{CD}_{2 n+2}, \mathrm{CF}[-\lambda],(t \wedge \leq k)\right)$, provided $n \cdot m-1 \geq(2 n+2) \cdot k$, i.e., $m \geq 2 \frac{n+1}{n} k+\frac{1}{n}$.

We now consider the other hierarchy, i.e., we fix $k$ and vary the number of components:

Corollary 10 Let $\Delta \in\{\leq,=\}$ and $k \in \mathbb{N}$ be fixed. The hierarchy of the families of languages $\mathscr{L}_{n}:=$ $\mathscr{L}\left(\mathrm{CD}_{n}, \mathrm{CF}[-\lambda],(t \wedge \Delta k)\right)$ with respect to $n \in \mathbb{N}$ is infinite, i.e., for every $n \in \mathbb{N}$ there exists an $m \in \mathbb{N}$, $m>n$, such that $\mathscr{L}_{n} \subset \mathscr{L}_{m}$.

Proof. We argue in a similar way as in the preceding corollary. First consider the $(t \wedge=k)$-mode of derivation. We already know that

$$
S_{m \cdot k} \in \mathscr{L}\left(\mathrm{CD}_{m+1}, \mathrm{CF}[-\lambda],(t \wedge=k+1)\right) .
$$

according to Theorem 7 , but $S_{m \cdot k} \notin \mathscr{L}_{m \cdot k-1}(\mathrm{P}, \mathrm{CF})$ according to Theorem 7 and, because of

$$
\mathscr{L}\left(\mathrm{CD}_{n}, \mathrm{CF}[-\lambda],(t \wedge=k+1)\right) \subseteq \mathscr{L}_{n \cdot(k+1)}(\mathrm{P}, \mathrm{CF})
$$

according to Theorem 6, $S_{m \cdot k}$ therefore cannot belong to $\mathscr{L}\left(\mathrm{CD}_{n}, \mathrm{CF}[-\lambda],(t \wedge=k+1)\right)$, provided $m$. $k-1 \geq n \cdot(k+1)$, i.e., $m \geq \frac{k+1}{k} n+\frac{1}{k}$.

By a similar reasoning, in case of the $(t \wedge \leq k)$-mode, for

$$
S_{m \cdot k} \in \mathscr{L}\left(\mathrm{CD}_{2 m+1}, \mathrm{CF}[-\lambda],(t \wedge \leq k+1)\right) \subseteq \mathscr{L}\left(\mathrm{CD}_{2 m+2}, \mathrm{CF}[-\lambda],(t \wedge \leq k+1)\right)
$$

we obtain $S_{m \cdot k} \notin \mathscr{L}\left(\mathrm{CD}_{2 n+1}, \mathrm{CF}[-\lambda],(t \wedge=k+1)\right) \cap \mathscr{L}\left(\mathrm{CD}_{2 n+2}, \mathrm{CF}[-\lambda],(t \wedge=k+1)\right)$, provided $m$. $k-1 \geq(2 n+2) \cdot(k+1)$, i.e., $m \geq 2 \frac{k+1}{k} n+\frac{2(k+1)}{k}\left(=2\left(1+\frac{1}{k}\right) n+2\left(1+\frac{1}{k}\right)\right)$.

In both cases, we see that the hierarchy with respect to the number of components is infinite.

Finally, let us consider the hierarchies for the "small cases" of $n$.

Lemma 11 Let $k \in \mathbb{N}$ and $\Delta \in\{\leq,=\}$.

$$
\begin{aligned}
\mathscr{L}\left(\mathrm{CD}_{1}, \mathrm{CF}[-\lambda],(t \wedge \Delta k)\right) & \subset \mathscr{L}\left(\mathrm{CD}_{2}, \mathrm{CF}[-\lambda],(t \wedge \Delta 1)\right) \\
& \subset \mathscr{L}\left(\mathrm{CD}_{3}, \mathrm{CF}[-\lambda],(t \wedge \Delta k)\right) .
\end{aligned}
$$

Proof. By our previous considerations, we know that $\mathscr{L}\left(\mathrm{CD}_{1}, \mathrm{CF}[-\lambda],(t \wedge \Delta k)\right)$ and $\mathscr{L}\left(\mathrm{CD}_{2}, \mathrm{CF}[-\lambda],(t \wedge \Delta 1)\right)$ coincide with $\mathscr{L}(\mathrm{FIN})$ and $\mathscr{L}(\mathrm{LIN})$, respectively, which already proves the first strict inclusion.

Now consider the non-linear language $\left\{a^{n} b^{n} a^{m} b^{m} \mid n, m \in \mathbb{N}\right\}$, which is generated by a CD grammar system

$$
G=\left(\left\{S, A, B, A^{\prime}, B^{\prime}\right\},\{a, b\}, S, P_{1}, P_{2}, P_{3}\right)
$$

taking $k=1$ with the following three components:

$$
\begin{aligned}
& P_{1}=\left\{S \rightarrow A B, A^{\prime} \rightarrow A, B^{\prime} \rightarrow B\right\} \\
& P_{2}=\left\{A \rightarrow a A^{\prime} b, A \rightarrow a b, B^{\prime} \rightarrow B^{\prime}\right\} \\
& P_{3}=\left\{B \rightarrow a B^{\prime} b, B \rightarrow a b, A \rightarrow A, A^{\prime} \rightarrow A^{\prime}\right\} .
\end{aligned}
$$


First, $P_{1}$ and $P_{2}$ have to be applied in sequence, say $n$ times, until $P_{2}$ uses the rule $A \rightarrow a b$. Now, $P_{3}$ can be applied. Then, $P_{1}$ and $P_{3}$ must be applied in sequence, say $m-1$ times, until $P_{3}$ terminates the whole derivation using $B \rightarrow a b$. In this way, a word $a^{n} b^{n} a^{m} b^{m}$ is derived. By the prolongation technique, the claimed assertion follows for $k>1$.

We conclude this section by remarking that the results presented in this section (originally contained in the Technical Report [7]) have been employed to show the following theorem in [1] that nicely complements our results here; we state these below with the notations of our paper.

Theorem 12 Let $n, k \geq 1$. Then we have

1. $\mathscr{L}\left(\mathrm{CD}_{n}, \mathrm{CF}[-\lambda],(t \wedge=k)\right) \subset \mathscr{L}\left(\mathrm{CD}_{n+2}, \mathrm{CF}[-\lambda],(t \wedge=k+1)\right)$ and

2. $\mathscr{L}\left(\mathrm{CD}_{n}, \mathrm{CF}[-\lambda],(t \wedge \leq k)\right) \subset \mathscr{L}\left(\mathrm{CD}_{2 \cdot(n+1)}, \mathrm{CF}[-\lambda],(t \wedge \leq k+1)\right)$.

\section{CD Grammar Systems and Programmed Grammars of Finite Index}

In this section we consider the finite index property for CD and HCD grammar systems and how they relate to programmed grammars of finite index in more detail.

Theorem 13 Let $m \in \mathbb{N}, F I=\{t\} \cup\left\{=m^{\prime \prime}, \geq m^{\prime \prime},\left(\geq m^{\prime \prime} \wedge \leq m^{\prime}\right),(t \wedge=k),(t \wedge \leq k),(t \wedge \geq k) \mid\right.$ $\left.m^{\prime}, m^{\prime \prime}, k \in \mathbb{N}, m^{\prime} \geq m^{\prime \prime} \geq m\right\}$, and $F$ contain all the hybrid modes considered in this paper, i.e., $F=\{(t \wedge=k),(t \wedge \leq k) \mid k \in \mathbb{N}\}$. Let $f \in F I$. Then

$$
\begin{aligned}
\mathscr{L}_{m}(\mathrm{P}, \mathrm{CF}[-\lambda]) & =\mathscr{L}_{m}\left(\mathrm{CD}_{\infty}, \mathrm{CF}[-\lambda], f\right) \\
& =\mathscr{L}_{m}\left(\mathrm{HCD}_{\infty}, \mathrm{CF}[-\lambda], F\right) .
\end{aligned}
$$

Proof. Looking through all the proofs showing the containment of HCD languages within programmed languages with appearance checking, it is easily seen that all these constructions preserve a finite index restriction.

Hence, it only remains to show that

$$
\mathscr{L}_{m}(\mathrm{P}, \mathrm{CF}[-\lambda]) \subseteq \mathscr{L}_{m}\left(\mathrm{CD}_{\infty}, \mathrm{CF}[-\lambda], f\right)
$$

for every $f \in F I$. Let $L \in \mathscr{L}_{m}(\mathrm{P}, \mathrm{CF})$ be generated by a programmed grammar $G=(N, T, P, S, \Lambda, \sigma)$ in NSF. Especially, there exists a function $f: \Lambda \rightarrow \underset{0}{N}$ such that, if $S \stackrel{*}{\Rightarrow} \underset{p}{\Rightarrow} w$ is a derivation in $G$, then $(f(p))(A)=|v|_{A} \leq 1$ for every nonterminal $A$.

We construct a simulating CD grammar system $G^{\prime}$ given by

$$
\left((N \times \Lambda) \cup(\{i \in \mathbb{N} \mid i \leq m\} \times N), T,(1, S),\left\{P_{I}\right\} \cup\left\{P_{p, q} \mid p \in \Lambda \wedge q \in \sigma(p)\right\}\right)
$$

of index $m$ all of whose components are working in one of the modes $=m, \geq m, t,\left(\geq m, \leq m^{\prime}\right)$ (with $\left.m^{\prime} \geq m\right),(t \wedge=m),(t \wedge \leq m)$, or $(t \wedge \geq m)$. Consider a production $\Lambda(p)=A \rightarrow w$ of $G$. We can assume (check) that $(f(p))(A)=1$. Furthermore, define

$$
n:=\sum_{B \in N}(f(p))(B) \leq m
$$

is the number of nonterminals in the current string (within a possible derivation leading to an application of $p$ ). 
Let the homomorphism $h_{p, q}:(N \times\{p\} \cup T)^{*} \rightarrow(N \times\{q\} \cup T)^{*}$ be defined by $(A, p) \mapsto(A, q)$ for $A \in N, a \mapsto a$ for $a \in T$.

For every $q \in \sigma$, we introduce a component $P_{p, q}$ within the CD grammar system containing the following productions:

If $n=m$, then $(A, p) \rightarrow h_{p, q}(w)$ simulates the (successful) application of rule $p$. If $n<m$, then we prolong the derivation in the following way:

$$
(A, p) \rightarrow(1, A), \quad(1, A) \rightarrow(2, A), \quad \ldots, \quad(m-n, A) \rightarrow h_{p, q}(w) .
$$

$(B, p) \rightarrow h_{p, q}(B)$ for $B \in N \backslash\{A\}$ keeps track of the information of the current state.

As initialization component, we take $P_{I}$ containing

$$
(1, S) \rightarrow(2, S), \quad \ldots, \quad(m, S) \rightarrow(S, p)
$$

for every $p \in \Lambda$ such that $(f(p))(S)=1$.

Observe that, by induction, to every sentential form derivable from the initial symbol $(1, S)$, any component applied to it can make either 0 steps (so, we selected the wrong one) or exactly $m$ steps.

By a simple prolongation trick, we can also take components working in one of the modes $=m^{\prime \prime}$, $\geq m^{\prime \prime},\left(\geq m^{\prime \prime}, \leq m^{\prime}\right)$ (with $\left.m^{\prime} \geq m^{\prime \prime}\right)$, for some $m^{\prime \prime} \geq m$.

Since the construction given in Theorem 2 is index-preserving, we can also take arbitrary $(t \wedge=k)$ or $(t \wedge \leq k)$ components instead of requiring $k \geq m$. Since the $t$-mode and the $(t \wedge \geq 1)$-mode are identical, the prolongation technique delivers the result for the $(t \wedge \geq k)$-mode for $k \in \mathbb{N}$ in general.

Our theorem readily implies a characterization of programmed languages of general finite index. We summarize this fact together with results obtained via a different simulation in [3, Theorem 3.26] in the following corollary.

Corollary 14 Let $m, k, k^{\prime} \in \mathbb{N}, k \geq 2$, and $\Delta \in\{\leq,=, \geq\}$. Then following families of languages coincide with $\mathscr{L}_{\text {fin }}(\mathrm{P}, \mathrm{CF}[-\lambda])$ :

1. $\mathscr{L}_{\text {fin }}\left(\mathrm{CD}_{\infty}, \mathrm{CF}[-\lambda], t\right)$,

2. $\mathscr{L}_{\text {fin }}\left(\mathrm{CD}_{\infty}, \mathrm{CF}[-\lambda],=k\right)$,

3. $\mathscr{L}_{\text {fin }}\left(\mathrm{CD}_{\infty}, \mathrm{CF}[-\lambda], \geq k\right)$,

4. $\mathscr{L}\left(\mathrm{CD}_{\infty}, \mathrm{CF}[-\lambda],(t \wedge=k)\right)$,

5. $\mathscr{L}\left(\mathrm{CD}_{\infty}, \mathrm{CF}[-\lambda],(t \wedge \leq k)\right)$, and

6. $\mathscr{L}_{\text {fin }}\left(\mathrm{CD}_{\infty}, \mathrm{CF}[-\lambda],\left(t \wedge \Delta k^{\prime}\right)\right)$.

As regards the number of components, we can state the following:

Theorem 15 Let $m \in \mathbb{N}, \Delta \in\{\leq,=, \geq\}$. Then we have
1. $\mathscr{L}_{m}\left(\mathrm{CD}_{\infty}, \mathrm{CF}[-\lambda], \Delta m\right)=\mathscr{L}_{m}\left(\mathrm{CD}_{3}, \mathrm{CF}[-\lambda], \Delta m\right)$ and
2. $\mathscr{L}_{m}\left(\mathrm{CD}_{\infty}, \mathrm{CF}[-\lambda],(t \wedge \Delta m)\right)=\mathscr{L}_{m}\left(\mathrm{CD}_{3}, \mathrm{CF}[-\lambda],(t \wedge \Delta m)\right)$.

Proof. Since we restrict our attention to languages of index $m$, we can simply carry over the proofs of the type "three is enough" for $t$-mode components, see [3, Theorem 3.10] and [13, Lemma 2]. 
In case of the $(t \wedge=m)$-mode, we have to go back to the simulation of the programmed grammar given in the preceding theorem. It is clear that, due to the nonterminal separation form (NSF) (see Theorem 1), we can prolong the simulation of a single nonterminal symbol.

It is quite natural to compare the families of languages defined by $\mathrm{CD}$ grammar systems obtained via the restriction of being of finite index $m$ with their unrestricted counterparts. In our case, it is interesting to see that also these unrestricted counterparts deliver languages of finite index. However, as we have seen in Theorem 8 ,

$$
S_{n \cdot k} \in \mathscr{L}\left(\mathrm{CD}_{n+1}, \mathrm{CF}[-\lambda],(t \wedge=k+1)\right)
$$

Especially, we have

$$
S_{2 \cdot(m-1)} \in \mathscr{L}\left(\mathrm{CD}_{3}, \mathrm{CF}[-\lambda],(t \wedge=m)\right) .
$$

Since $S_{2 \cdot(m-1)}$ is not of (programmed) index $2 m-3$, we can state:

Corollary 16 For $m \in \mathbb{N}$, we have

$$
\mathscr{L}_{m}\left(\mathrm{CD}_{3}, \mathrm{CF}[-\lambda],(t \wedge=m)\right) \subset \mathscr{L}\left(\mathrm{CD}_{3}, \mathrm{CF}[-\lambda],(t \wedge=m)\right) .
$$

Proof. Our previous considerations deliver the case $m>2$, since $S_{2(m-1)}$ is not a (programmed) language of index $2 m-3$, and therefore not a (programmed) language of index $m$; hence,

$$
S_{2 \cdot(m-1)} \notin \mathscr{L}_{m}\left(\mathrm{CD}_{3}, \mathrm{CF}[-\lambda],(t \wedge=m)\right) .
$$

In case $m=2$, we know that

$$
S_{3} \notin \mathscr{L}_{2}\left(\mathrm{CD}_{3}, \mathrm{CF}[-\lambda],(t \wedge=2)\right) .
$$

On the other hand, the $\left(\mathrm{CD}_{3}, \mathrm{CF}[-\lambda],(t \wedge=2)\right)$ grammar system

$$
G=\left(\left\{S, A, B, A^{\prime}, B^{\prime} C, C^{\prime}, B^{\prime \prime}, F\right\},\{a, b\}, S, P_{1}, P_{2}, P_{3}\right)
$$

with the following three components generates $S_{3}$, starting with $S$ :

$$
\begin{aligned}
& P_{1}=\left\{A \rightarrow a A^{\prime} a, A \rightarrow a b a, B \rightarrow a B^{\prime} a, B \rightarrow B^{\prime \prime}\right\} \\
& P_{2}=\left\{B^{\prime} \rightarrow B, C \rightarrow a C^{\prime} a, A \rightarrow F, B^{\prime \prime} \rightarrow a b a, C \rightarrow a b a\right\} \\
& P_{3}=\left\{S \rightarrow b A b B b C b, C^{\prime} \rightarrow C, A^{\prime} \rightarrow A, B^{\prime} \rightarrow F\right\} .
\end{aligned}
$$

Finally, we have $\mathscr{L}_{1}\left(\mathrm{CD}_{3}, \mathrm{CF}[-\lambda],(t \wedge=1)\right)=\mathscr{L}(\mathrm{LIN})$. The example $\left\{a^{n} b^{n} a^{m} b^{m} \mid n, m \in \mathbb{N}\right\} \notin$ $\mathscr{L}(\mathrm{LIN})$ in Lemma 11 was shown to be in $\mathscr{L}\left(\mathrm{CD}_{3}, \mathrm{CF}[-\lambda],(t \wedge=1)\right)$.

If we admit four components (or arbitrarily many), by a similar reasoning we can separate all corresponding classes, since $3(m-1)=3 m-3>m$ for $m \geq 2$.

Corollary 17 For $m, n \in \mathbb{N}, n \geq 4$ (or $n=\infty)$, we have

$$
\mathscr{L}_{m}\left(\mathrm{CD}_{n}, \mathrm{CF}[-\lambda],(t \wedge=m)\right) \subset \mathscr{L}\left(\mathrm{CD}_{n}, \mathrm{CF}[-\lambda],(t \wedge=m)\right) .
$$

Observe that the results exhibited in the last two corollaries are quite astonishing if one keeps in mind that

$$
\begin{aligned}
\mathscr{L}_{\text {fin }}(\mathrm{P}, \mathrm{CF}) & =\bigcup_{m \in \mathbb{N}} \mathscr{L}_{m}\left(\mathrm{CD}_{n}, \mathrm{CF}[-\lambda],(t \wedge=m)\right) \\
& =\bigcup_{m \in \mathbb{N}} \mathscr{L}\left(\mathrm{CD}_{n}, \mathrm{CF}[-\lambda],(t \wedge=m)\right)
\end{aligned}
$$

for all $n \in \mathbb{N}, n>2$. 


\section{Conclusions and Prospects}

In this paper we have studied CD grammar systems working in the internally hybrid modes $(t \wedge=m)$ and $(t \wedge \leq m)$ together with the finite index restriction. Showing specific relations to programmed grammars of finite index, we were able to establish infinite hierarchies for CD grammar systems of finite index working in the internally hybrid modes $(t \wedge=k)$ and $(t \wedge \leq k)$ both with respect to the number of components $n$ and the number of maximal steps $k$. However, many quite natural questions still remain open. For instance, Theorem 12 leaves open the strictness of several natural inclusion relations relating the parameters "numbers of components" $n$ and "step number bound" $k$.

It is well-known that ETOL systems are tightly related to CD grammar systems working in the $t$ mode. In the literature, several step-bound restrictions have been discussed in relation with parallel systems, see [5] for an overview. Are these somehow related to the internally hybrid systems discussed in this paper (and their companions)? Or do hybrid modes lead to new (natural) derivation modes for parallel systems? In particular, the finite index restriction studied in Section 5 could be of interest in this context, although (and also because) we are not aware of a study of finite index in the context of limited parallel rewriting, which might be an interesting research question in its own.

Acknowledgements Most of the research of the first and last author was undertaken while being affiliated to Wilhelm-Schickard Institut für Informatik, Universität Tübingen, Sand 13, D-72076 Tübingen, Germany. Part of the research of the first author was supported by Deutsche Forschungsgemeinschaft, grant DFG La 618/3-1/2 “Komplexitätstheoretische Methoden für die adäquate Modellierung paralleler Berechnungen."

\section{References}

[1] H. Bordihn \& M. Holzer (1999): On a hierarchy of languages generated by cooperating distributed grammar systems. Information Processing Letters 69(2), pp. 59-62, doi:10.1016/S0020-0190(98)00200-2

[2] E. Csuhaj-Varjú \& J. Dassow (1990): On cooperating/distributed grammar systems. J. Inf. Process. Cybern. EIK (formerly Elektron. Inf.verarb. Kybern. 26(1/2), pp. 49-63.

[3] E. Csuhaj-Varjù, J. Dassow, J. Kelemen \& Gh. Păun (1994): Grammar Systems. A Grammatical Approach to Distribution and Cooperation. Gordon and Breach, London.

[4] J. Dassow \& Gh. Păun (1989): Regulated Rewriting in Formal Language Theory. EATCS Monographs in Theoretical Computer Science 18, Springer Berlin, doi 10.1007/978-3-642-74932-2

[5] H. Fernau (2003): Parallel Grammars: A Phenomenology. GRAMMARS 6, pp. 25-87, doi $10.1023 / \mathrm{A}: 1024087118762$.

[6] H. Fernau \& R. Freund (1996): Bounded parallelism in array grammars used for character recognition. In P. Perner, P. Wang \& A. Rosenfeld, editors: Advances in Structural and Syntactical Pattern Recognition (Proceedings of the SSPR'96), Lecture Notes in Computer Science 1121, Springer, pp. 40-49, doi:10.1007/3540-61577-6_5.

[7] H. Fernau, R. Freund \& M. Holzer (1996): External versus Internal Hybridization for Cooperating Distributed Grammar Systems. Technical Report TR 185-2/FR-1/96, Technische Universität Wien (Austria).

[8] H. Fernau, R. Freund \& M. Holzer (2001): Hybrid modes in cooperating distributed grammar systems: internal versus external hybridization. Theoretical Computer Science 259(1-2), pp. 405-426, doi:10.1016/S03043975(00)00022-0. 
[9] H. Fernau, R. Freund \& M. Holzer (2003): Hybrid modes in cooperating distributed grammar systems: combining the $t$-mode with the modes $\leq k$ and $=k$. Theoretical Computer Science 299, pp. 633-662, doi $10.1016 / \mathrm{S} 0304-3975(02) 00541-8$.

[10] H. Fernau \& M. Holzer (1996): Accepting multi-agent systems II. Acta Cybernetica 12, pp. 361-379.

[11] H. Fernau \& M. Holzer (1997): Conditional context-free languages of finite index. In Gh. Păun \& A. Salomaa, editors: New Trends in Formal Languages, Lecture Notes in Computer Science 1218, Springer, pp. 10-26, doi $10.1007 / 3-540-62844-4 \_2$.

[12] R. Meersman \& G. Rozenberg (1978): Cooperating grammar systems. In: Proceedings of Mathematical Foundations of Computer Science MFCS'78, Lecture Notes in Computer Science 64, Springer, pp. 364-374, doi $10.1007 / 3-540-08921-7 \_84$.

[13] V. Mitrana (1993): Hybrid cooperating/distributed grammar systems. Computers and Artificial Intelligence 12(1), pp. 83-88.

[14] Gh. Păun (1994): On the generative capacity of hybrid CD grammar systems. J. Inf. Process. Cybern. EIK (formerly Elektron. Inf.verarb. Kybern.) 30(4), pp. 231-244.

[15] G. Rozenberg \& A. Salomaa, editors (1997): Handbook of Formal Languages, 3 volumes. Springer, Berlin.

[16] G. Rozenberg \& D. Vermeir (1978): On the effect of the finite index restriction on several families of grammars; Part 2: context dependent systems and grammars. Foundations of Control Engineering 3(3), pp. $126-142$. 